\title{
AZD3463, an IGF-1R inhibitor, suppresses breast cancer metastasis to bone via modulation of the PI3K-Akt pathway
}

\author{
Guang-Fu Hu ${ }^{1 \#}$, Cheng Wang ${ }^{1 "}$, Guang-Xia $\mathrm{Hu}^{2}$, Ge Wu ${ }^{2}$, Chengjiao Zhang ${ }^{3}$, Wei Zhu ${ }^{4}$, Cong Chen ${ }^{4}$, \\ Yutong $\mathrm{Gu}^{5,6}$, Hongwei Zhang ${ }^{4}$, Zi'ang Yang ${ }^{4}$
}

${ }^{1}$ Department of Breast Surgery, Huangpu Branch, Shanghai Ninth People's Hospital, Affiliated to Shanghai Jiao Tong University School of Medicine, Shanghai 200011, China; ${ }^{2}$ Department of Pathology, Binzhong People's Hospital, Binzhong 256600, China; ${ }^{3}$ Department of Psychological Measurement, Shanghai Mental Health Center, Shanghai Jiao Tong University School of Medicine, Shanghai 20030, China; ${ }^{4}$ Department of General Surgery, ${ }^{5}$ Department of Orthopaedic Surgery, Zhongshan Hospital Fudan University, Shanghai 200032, China; ${ }^{6}$ Department of Orthopaedic Surgery, Shanghai Public Health Clinical Center, Fudan University, Shanghai 201508, China

Contributions: (I) Conception and design: GF Hu, C Wang, Z Yang, H Zhang, Y Gu; (II) Administrative support: None; (III) Provision of study materials or patients: None; (IV) Collection and assembly of data: GF Hu, C Wang, GX Hu, G Wu, C Zhang, W Zhu, C Chen; (V) Data analysis and interpretation: GF Hu, C Wang; (VI) Manuscript writing: All authors; (VII) Final approval of manuscript: All authors.

\#These authors contributed equally to this work.

Correspondence to: Zi'ang Yang; Hongwei Zhang. Department of General Surgery, Zhongshan Hospital Fudan University, 180 Fenglin Road, Shanghai 200032, China. Email: yang.zi_ang@zs-hospital.sh.cn; zhang.hongwei@zs-hospital.sh.cn; Yutong Gu. Department of Orthopaedic Surgery, Zhongshan Hospital Fudan University, 180 Fenglin Road, Shanghai 200032, China; Department of Orthopaedic Surgery, Shanghai Public Health Clinical Center, Fudan University, Shanghai 201508, China. Email: gu.yutong2@zs-hospital.sh.cn.

Background: The bone-derived insulin-like growth factor I (IGF-1) and its receptor IGF-1R play a crucial role in promoting the survival and proliferation of cancer cells, and have thus been considered as prime targets for the development of novel antitumor therapeutics.

Methods: By using the MDA-MB-231BO cell line, which is the osteotropic metastatic variant of the human breast adenocarcinoma cell line MDA-MB-231, and an in vivo model of breast cancer metastasis to bone, the current study evaluated the effect of AZD3463, an IGF-1R inhibitor, used alone or in combination with zoledronic acid (ZA), on the regulation of IGF-1R associated signal pathway and treatment of bone metastases (BM). Cell proliferation and invasion were measured by methyl thiazolyl tetrazolium (MTT) and Transwell assay respectively. Apoptotic cell number was detected by terminal deoxynucleotidyl transferasemediated dUTP-biotin nick end labeling (TUNEL).

Results: AZD3463 was shown to alleviate IGF-1R phosphorylation promoted by IGF-1 treatment in MDA-MB-231BO cells in a dose-dependent manner. In both the cells and the mouse model, $5 \mathrm{nM}$ of AZD3463 stimulated cell apoptosis and suppressed proliferation on a level similar to that of $100 \mu M$ of ZA. Remarkably, the combined use of AZD3463 and ZA exhibited a synergistic effect and greater antitumor activity compared to when they were employed individually. Mechanistic investigations indicated that the apoptosis-inducing activity of AZD3463 could be associated to its role in the activation of the phosphoinositide 3-kinase (PI3K)-Akt signaling pathway.

Conclusions: These findings suggested that AZD3463 could serve as a promising therapeutic molecule for treating BM in breast cancer patients, particularly when applied in conjunction with ZA or other antitumor agents.

Keywords: Insulin-like growth factor I receptors (IGF-1R); bone metastases (BM); breast cancer; phosphoinositide 3-kinase-Akt pathway (PI3K-Akt pathway)

Submitted Dec 21, 2019. Accepted for publication Feb 06, 2020.

doi: 10.21037/atm.2020.02.110

View this article at: http://dx.doi.org/10.21037/atm.2020.02.110 


\section{Introduction}

Metastatic breast cancer is the leading cause of cancerrelated deaths among women $(1,2)$. More than $70 \%$ of patients with advanced breast cancer develop bone metastases $(\mathrm{BM})$, which result in high morbidity and mortality rates (3), as well as severely impaired quality of life $(4,5)$. In addition, breast cancer patients with BM are extremely susceptible to bone-related complications, also known as skeletal-related events (SREs), that require medical and surgical interventions. Common forms of SREs include pain, pathological fractures, spinal cord compression and hypercalcemia $(6,7)$. Currently, bisphosphonates and the recently developed monoclonal antibody (mAb) denosumab are the most commonly used therapeutic agents for managing $\mathrm{BM}$ derived from breast cancer. However, it has been suggested that neither provides any survival advantage $(8,9)$. Furthermore, the optimal duration and treatment schedule of these drugs are often not defined. It has been suggested that the development of a novel therapeutic agent with a mechanism of action distinct from that of bisphosphates would provide considerable benefits to the management of $\mathrm{BM}$ in breast cancer patients.

Interactions between cancer cells and the microenvironment at the site of metastasis play an important role for the development of secondary tumors (10). In the case of BM, improved understanding of its underlying mechanisms could greatly contribute to the invention of new, effective therapeutic strategies. Studies have shown that osteotropic tumors can secrete parathyroid hormone-related peptide, leading to stimulation of osteoclastogenesis (11). Similarly, bone stroma produces various growth factors, such as transforming growth factor- $\beta$, that promote tumor growth in bone (12). Therapeutic targeting of these microenvironmental factors is under intensive investigation. Other attractive targets include receptor activator of nuclear factor $\kappa \mathrm{B}(\mathrm{NF}-\mathrm{\kappa B})$ ligand, Src kinase and cathepsin $\mathrm{K}$, all of which are involved in the regulation of osteoclast functions, as well as chemokine receptor 4, which is closely involved in the high propensity of certain cancers toward bone migration (13).

Recently, Hiraga et al. reported that the bone-derived insulin-like growth factor I (IGF-1) promoted BM of human breast cancer cells through its stimulation of cell proliferation and inhibition of apoptosis (14). Association of IGF-1 with IGF-1 receptors (IGF-1R) were shown to activate both the serine/threonine kinase Akt and the transcription factor $\mathrm{NF}-\kappa \mathrm{B}$, resulting in increased cell survival $(15,16)$. In contrast, disrupting the activation of IGF-1R, Akt or NF- $\kappa \mathrm{B}$ could significantly inhibit BM.
The results from previous studies strongly suggested that the IGF-1R signaling pathway, whose activation by bone-derived IGF-1 contributed mechanistically to the development of BM in breast cancer patients, could serve as a potential therapeutic target. Recently, AZD3463 was developed by AstraZeneca as a potent inhibitor of anaplastic lymphoma kinase, IGF-1R and additional kinases for clinical use against crizotinib-resistant anaplastic large cell lymphoma and small cell lung cancer (17). Herein we report the evaluation of AZD3463, an IGF-1R inhibitor, administered alone or in combination with zoledronic acid (ZA), for the treatment of breast cancer-derived BM using both an osseous metastatic variant of human breast adenocarcinoma cell line and a murine BM model.

\section{Methods}

\section{Reagents and chemicals}

AZD3463 (AstraZeneca UK Limited, London, England) was dissolved in $10 \%$ DMSO to a final concentration of $10 \mathrm{nM}$ for both in vitro and in vivo experiments. ZA was provided by Novartis China, Beijing, China. IGF-I was obtained from BioVision, San Francisco, CA, USA. CK7 antibody was purchased from Abcam, Cambridge, UK. All other chemicals and reagents used in this study were purchased from Sigma-Aldrich, St. Louis, MO, USA unless noted otherwise.

\section{Cell lines and treatment experiments}

The osteotropic metastatic variant of MDA-MB-231, MDA-MB-231BO, was kindly provided by Dr. Toshiyuki Yoneda at the University of Texas Health Science Center at San Antonio, San Antonio, Texas, USA. The cells were maintained at $37^{\circ} \mathrm{C}$ in Dulbecco's Modified Eagle Medium (DMEM; Life Technologies, Grand Island, NY, USA) supplemented with $10 \%$ fetal bovine serum (FBS; Hyclone Laboratories, Logan, UT, USA) and $1 \%$ penicillinstreptomycin solution (Life Technologies, Grand Island, NY, USA) under a humidified atmosphere with $5 \% \mathrm{CO}_{2}$ in air. To determine the optimal duration for IGF-1 treatment, MDA-MB-231BO cells were serum starved overnight and then incubated with $100 \mathrm{ng} / \mathrm{mL}$ IGF-1 for varying periods of time, including $10 \mathrm{~min}, 30 \mathrm{~min}, 2 \mathrm{~h}, 8 \mathrm{~h}$ and $24 \mathrm{~h}$. To determine the optimal concentration of AZD3463, cells were co-incubated with $100 \mathrm{ng} / \mathrm{mL} \mathrm{IGF-1} \mathrm{and} 0.5,2,5$ or $10 \mathrm{nM}$ of AZD3463 for $24 \mathrm{~h}$. To study the effect of AZD3463 on the phosphorylation of IGF-1R downstream 
targets and on MDA-MB-231BO cell viability, cells were divided into five experiment groups, including a negative control group, a positive control group (IGF-1 treatment), an AZD3463 group, a ZA group and an AZD3463/ZA group. All groups except the negative control group were treated with $100 \mathrm{ng} / \mathrm{mL} \mathrm{IGF-1} \mathrm{at} \mathrm{for} 24 \mathrm{~h}$. In addition, the AZD3463 group, ZA group and AZD3463/ZA group were co-incubated with $5 \mathrm{nM}$ AZD3463, $100 \mu \mathrm{M} \mathrm{ZA}$ and both, respectively.

\section{Western blotting}

Western blotting was performed as previously described (18-20). IGF-I Receptor $\beta$ (D23H3) XP Rabbit mAb, Phospho-IGF-I Receptor $\beta$ (Tyr1135/1136)/Insulin Receptor $\beta$ (Tyr1150/1151) (19H7) Rabbit mAb and Phospho-Akt Pathway Antibody Sampler Kit were purchased from Cell Signaling Technologies, College Park, MD, USA. Anti- $\beta$-actin antibody was purchased from Sigma-Aldrich, St. Louis, MO, USA.

\section{Methyl thiazolyl tetrazolium (MTT) assay and Transwell migration assay}

MTT assay and Transwell migration assay were performed based on previously described protocols $(18,21)$. For MTT assay, cells were trypsin-digested and then seeded to each well of a 96-well plate to a density of 500 cells per well. For Transwell assay, the digested cells were added to the upper Transwell chamber in $300 \mu \mathrm{L}$ serum-free DMEM. Migrated cells were fixed in $40 \%$ formalin for $30 \mathrm{~min}$ and stained with $0.1 \%$ crystal violet.

\section{Terminal deoxynucleotidyl transferase-mediated dUTP- biotin nick end labeling (TUNEL) assay}

Apoptotic breast cancer cells were detected by TUNEL assay using the fluorometric TUNEL system (Promega, Madison, WI, USA) according to the protocol provided by the manufacturer. Briefly, $50 \mu \mathrm{L}$ of rTdT incubation buffer was added to the permeabilized cells on slides, which were then incubated under humidified atmosphere for $60 \mathrm{~min}$ in darkness and rinsed three times with $2 \mathrm{X}$ saline-sodium citrate buffer. The slides were subsequently stained with DAPI and imaged under a fluorescence microscope (BX51, Olympus, Japan). TUNEL-positive cells were indicated by the emission of green fluorescence and the nuclei were indicated by their blue fluorescence.

\section{Establishment of the murine BM model and therapeutic intervention}

Female, 4-week-old, nude mice were maintained in the animal facility of Zhongshan Hospital Fudan University. All animal experiments were performed in accordance with the Guide for the Care and Use of Laboratory Animal of Zhongshan Hospital Fudan University and approved by the Ethics Committee. One week after intracardiac inoculation of MDAMB-231BO cells, BM development in mice was detected using the IVIS Lumina XR Series III In Vivo Imaging System (PerkinElmer, Waltham, MA, USA). A total of 24 mice were found to have developed $\mathrm{BM}$, which were then divided into four equal groups: a control group, an AZD3463 group, a ZA group and an AZD3463/ZA group. The animals in the four experiment groups were subjected to intraperitoneal injections of different reagents at varying frequencies. The control group were administered with $10 \%$ DMSO daily as a vehicle control for eight days. The AZD3463 group received AZD3463 at a dose of $15 \mathrm{mg} / \mathrm{kg}$ body weight once every 2 days for a total of four times. The mice of the ZA group were administered with $4 \mu \mathrm{g} /$ mouse ZA once a day over eight days, whereas those in the AZD3463/ZA group were subjected to a combination of ZA and AZD3463 treatment mentioned above. All mice were maintained under otherwise identical conditions, scanned by the IVIS Lumina XR Series III In Vivo Imaging System every week, and sacrificed on day 36 after the initial inoculation of the cancer cells.

\section{Histological analyses}

Following the sacrifice, hind limb long bones and vertebras are removed and fixed in phosphate-buffered saline (PBS, $\mathrm{pH} 7.2$ ) containing $10 \%$ formalin for 2 days, followed by decalcification in $14 \%$ ethylenediaminetetraacetic acid (EDTA) with constant stirring over 2 weeks. The decalcified bone specimens were then embedded in paraffin and stained with hematoxylin-eosin ( $\mathrm{H} \& \mathrm{E}$ ) based on previously described protocols $(20,21)$. For immunohistological staining, bone sections were first incubated with a primary antibody against CK7, an epithelial cell marker, and then incubated with a biotinylated secondary antibody after washing with PBS. Signal was generated by incubation with streptavidin-horseradish peroxidase complex.

\section{Statistical analyses}

All data were expressed as mean \pm standard error. 

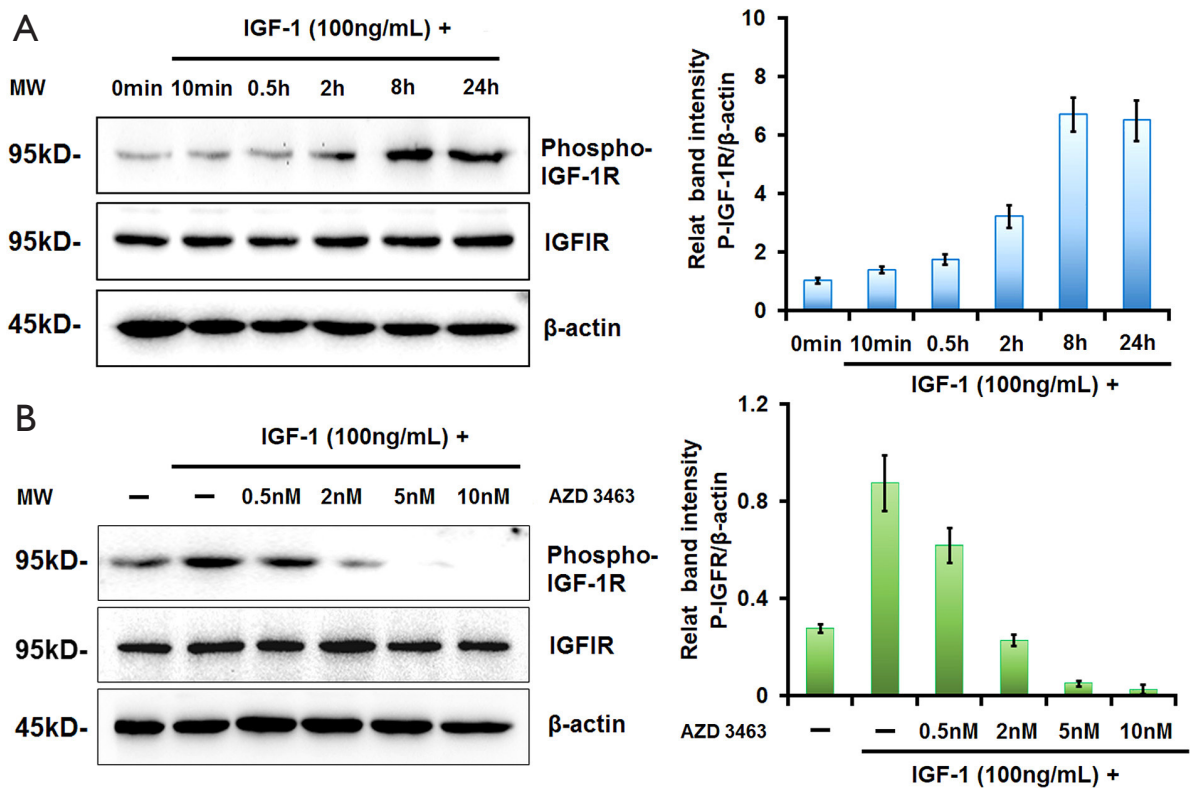

Figure 1 AZD3463 could attenuate IGF-1-promoted phosphorylation of IGF-1R in a dose-dependent manner. (A) Left: Western blot showing the levels of phosphorylated and total IGF-1R at various time points after IGF-1 exposure. Right: Western blot band intensities for Phospho-IGF-1R relative to $\beta$-actin were evaluated; (B) left: Western blot showing the levels of indicated proteins in the presence of a combination of $100 \mathrm{ng} / \mathrm{mL}$ of IGF-1 and varying concentrations of AZD3463. Right: Western blot band intensities for Phospho-IGF$1 \mathrm{R}$ relative to $\beta$-actin were evaluated. $\beta$-Actin was used as an internal control. The error bars represent the SD of the mean from three independent experiments. IGF-1, insulin-like growth factor I; IGF-1R, IGF-1 receptors.

Statistical analysis was performed using StatView (SAS Institute, Cary, NC, USA). Student $t$-test or Welch $t$-test was conducted to assess differences between any two experiment groups. One-way analysis of variance followed by Fisher's least significant difference post-hoc test was used for differences among three or more experiment groups. $\mathrm{P}<0.05$ (denoted by asterisks) was considered statistically significant.

\section{Results}

AZD3463 attenuated the IGF-1-promoted phosphorylation of IGF-1R and concomitantly enhanced the migration of MDA-MB-231BO cells

We began our study by confirming the stimulatory effect of IGF-1 on the proliferation of MDA-MB-231BO cells, which are the osteotropic variant of the human breast adenocarcinoma cell line MDA-MB-231 (22). Consistent with previous findings (14), Western blot analysis showed that incubation of MDA-MB-231BO cells with $100 \mathrm{ng} / \mathrm{mL}$ of IGF-1 resulted in a time-dependent increase of IGF-1R phosphorylation, which plateaued after $24 \mathrm{~h}$ (Figure 1A). Based on the data, we chose $24 \mathrm{~h}$ as the incubation time for IGF-1 stimulation in all subsequent cellular experiments. We next evaluated whether AZD3463, a preclinical IGF$1 \mathrm{R}$ inhibitor, could reverse the phosphorylation of IGF-1R induced by its association with IGF-1. Based on a previous study that calculated the $\mathrm{IC}_{50}$ of AZD3463 to be in the range of 2.8 to $21.3 \mathrm{nM}$ in wild-type ALK neuroblastoma cells (17), we treated MDA-MB-231BO cells with both $100 \mathrm{ng} / \mathrm{mL}$ of IGF-1 and varying concentrations of AZD3463 in the range of $0.5-10 \mathrm{nM}$ for $24 \mathrm{~h}$. Compared to the IGF-1-treated cells, incubation with AZD3463 reduced the level of phosphorylated IGF-1R in a dose-dependent manner, with the total amount of the receptor remaining relatively stable (Figure 1B).

Consistent with the previous findings $(15,16)$, MTT assay demonstrated that IGF-1 treatment resulted in a 3 -fold increase in the number of viable MDA-MB$231 \mathrm{BO}$ cells over $96 \mathrm{~h}$ compared to the mock experiment in which no IGF-1 was added (Figure $2 A$ ). However, coincubation with AZD3463 mitigated the elevation of cell viability in a dose-dependent manner, particularly 


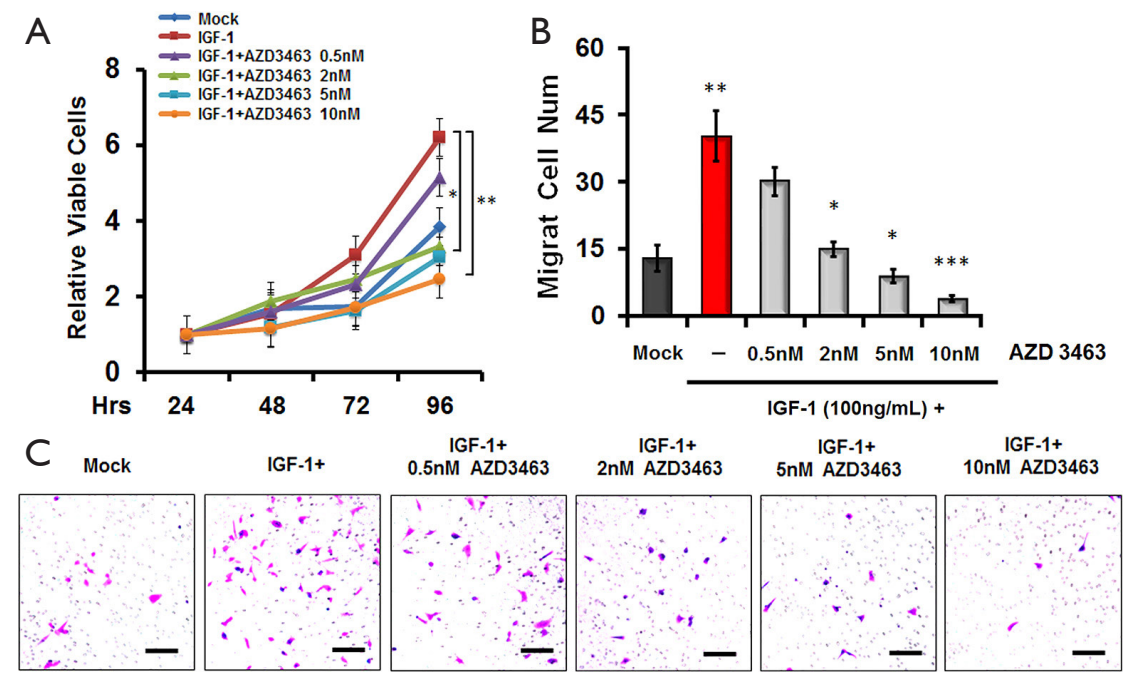

Figure 2 AZD3463 could reduce the proliferation and migration of MDA-MB-231BO cells in a dose-dependent manner. (A) MTT assay showing the relative viable cell numbers in different experiment groups; (B) Transwell assay indicating a gradual decline in the number of migrated MDA-MB-231BO cells with increasing AZD3463 concentrations; (C) crystal violet staining images of migrating cells. Scale bar, $100 \mu \mathrm{m} .{ }^{*}, \mathrm{P}<0.05 ;{ }^{* *}, \mathrm{P}<0.01 ;{ }^{* * *}, \mathrm{P}<0.001$. MTT, methyl thiazolyl tetrazolium; IGF-1, insulin-like growth factor I.

at 5 and $10 \mathrm{nM}$. These findings were echoed by the cell invasion assay, in which the migration of MDA-MB$231 \mathrm{BO}$ cells were stimulated by IGF-1 but suppressed in a dose-dependent fashion by the inhibitor $\left({ }^{*}, \mathrm{P}<0.05\right.$; **, $\mathrm{P}<0.01$; ***, $\mathrm{P}<0.001$ ) (Figure $2 B, C)$. Notably, cells treated with $100 \mathrm{ng} / \mathrm{mL}$ of IGF-1 and $10 \mathrm{nM}$ of AZD3463 exhibited $87 \%$ lower invasive capabilities than those with IGF-1 stimulation. Based on the experimental data, we opted to set the concentration of AZD3463 to $5 \mathrm{nM}$ in all subsequent experiments. In addition, the results confirmed that AZD3463 could alleviate the stimulatory effect of IGF-1 on the viability of MDA-MB-231BO cells by preventing IGF-1R phosphorylation.

\section{Combination of AZD3463 and $Z A$ suppresses the viability, proliferation and migration of $M D A-M B-231 B O$ cells}

$\mathrm{ZA}$ is one of the most commonly used bisphosphate compounds that effectively inhibits bone resorption and ameliorates osteoporosis. In recent years, ZA has also been employed to reduce the risk of bone fractures in patients with $\mathrm{BM}$ (23). As a result, we decided to compare the effects of AZD3463 and ZA on the proliferative and metastatic capabilities of MDA-MB-231BO cells. Both $100 \mu \mathrm{M}$ of $\mathrm{ZA}$ and $5 \mathrm{nM}$ of AZD3463 were found to completely abolish the IGF-1-induced increase of cell viability as indicated by the MTT assay (Figure $3 A$ ) and the cell invasion assay (Figure $3 B, C$ ), respectively. Furthermore, the combined use of both ZA and AZD3463 resulted in a further, statistically significant decrease in the invasive ability of MDA-MB-231BO cells compared to when only one of the therapeutic agent was included $\left({ }^{*}, \mathrm{P}<0.05 ;{ }^{* *}, \mathrm{P}<0.01\right.$ ) (Figure $\left.3 A, B, C\right)$. Consistent with these results, Tunel assay showed that the percentages of apoptotic MDA-MB-231BO cells in the IGF-1/AZD3463treated group and in the group co-incubated with IGF-1 and $\mathrm{ZA}$ were $21.2 \%$ and $58.6 \%$, respectively, up from $3.3 \%$ when the cells were only treated with IGF-1, or $8.5 \%$ in the untreated control group (Figure 4A,B). Remarkably, the combined application of both therapeutic agents induced apoptosis in $87.7 \%$ of the total cell population after $24 \mathrm{~h}$ of incubation (Figure $4 A, B$ ).

\section{AZD3463 blocks IGF-1 signaling pathway by suppressing the phosphorylation of Akt}

Previous studies have indicated that IGF-1 could regulate cell apoptosis by activating the phosphoinositide 3-kinase (PI3K)-Akt signaling pathway and/or the Raf-MAPK/ ERK kinase (MEK)-extracellular regulated kinase (ERK) signaling pathway (24). To probe the mechanistic role of AZD3463 in the induction of MDA-MB-231BO cell apoptosis, we analyzed the expression levels of various factors involved in the PI3K-Akt pathway and the MEK- 

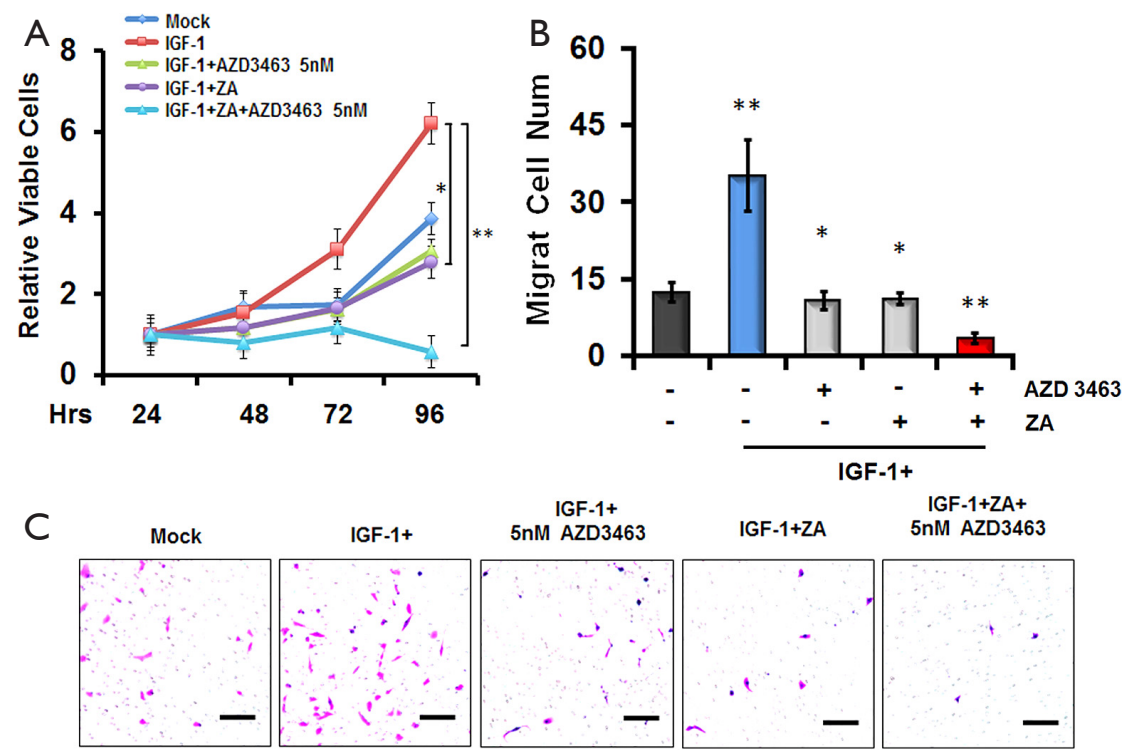

Figure 3 The combination of AZD3463 and ZA suppressed the proliferation and migration of MDA-MB-231BO cells. (A) MTT assay showing the relative viable cell numbers in different experiment groups; (B) Transwell assay indicating the numbers of migrated cells in different experiment groups; (C) crystal violet staining images of migrating cells. Scale bar, $100 \mu \mathrm{m}$. *, $\mathrm{P}<0.05$; **, $\mathrm{P}<0.01$. ZA, zoledronic acid; MTT, methyl thiazolyl tetrazolium; IGF-1, insulin-like growth factor I.

ERK pathway. As illustrated in Figure 4C, both AZD3463 and ZA could reverse IGF-1-promoted phosphorylation of IGF-1R, with their combination exhibiting a further augmented effect. Compared to the untreated control group, cells incubated with IGF-1 showed enhanced phosphorylation of Akt, Raf and similar phosphorylation levels of glycogen synthase kinase 3 beta (GSK-3 $\beta$ ) and phosphatase and tensin homolog (PTEN) (Figure 4C). Treatment with AZD3463 led to a significant drop in Akt phosphorylation but no obvious change in the levels of the other proteins tested (Figure 4C). Meanwhile, cells incubated with ZA exhibited a further decrease of Akt phosphorylation, and noticeably, an elevation in the level of phosphorylated PTEN, with no detectable impact on Raf or GSK-3 $\beta$ phosphorylation (Figure $4 C$ ). It should be emphasized that the observed regulatory effects of $\mathrm{ZA}$ on Akt and PTEN were consistent with the results described in several previously published studies (25-27). Finally, the combination of AZD3463 and ZA also caused the level of phosphorylated Akt to decline and that of phosphorylated PTEN to further increase, without exerting any observable effect on the phosphorylation of Raf or GSK-3 $\beta$ (Figure $4 C$ ). Taken together, the experimental data suggested that AZD3463 modulated the apoptosis of MDAMB-231BO cells through its activation of the PI3K-Akt signaling pathway.

\section{AZD 3463, alone or coupled with $\mathrm{ZA}$, could prevent the metastasis of breast cancer to bone}

To further assess the clinical relevance of AZD3463, we generated a murine model of breast cancer metastasis to bone and investigated the effectiveness of different treatment strategies against $\mathrm{BM}$ in vivo (Figure $5 A, B, C, D$ ). After the intracardiac injection of MDA-MB-231BO cells, mice showing signs of $\mathrm{BM}$ development were divided into four experiment groups, each of which were administered with physiological saline, AZD3463, ZA or a combination of both drugs. The mice treated with physiological saline (control group) were indicated by bioluminescent imaging (BLI) to have developed multiple BM one week following the injection of cancer cells (Figure $5 A, B$ ). At 5 weeks, the tumor burden in these mice showed a dramatic increase. In comparison, both the ZA group and the AZD3463 group displayed fewer and smaller tumor lesions compared to the control throughout the course of the treatment (Figure 5B). Quantitation of BLI revealed that the use of ZA reduced the tumor burden by $38.4 \% 5$ weeks after the injection of MDA-MB-231BO cells, whereas AZD3463 was found, around the same period, to result in a $69 \%$ 

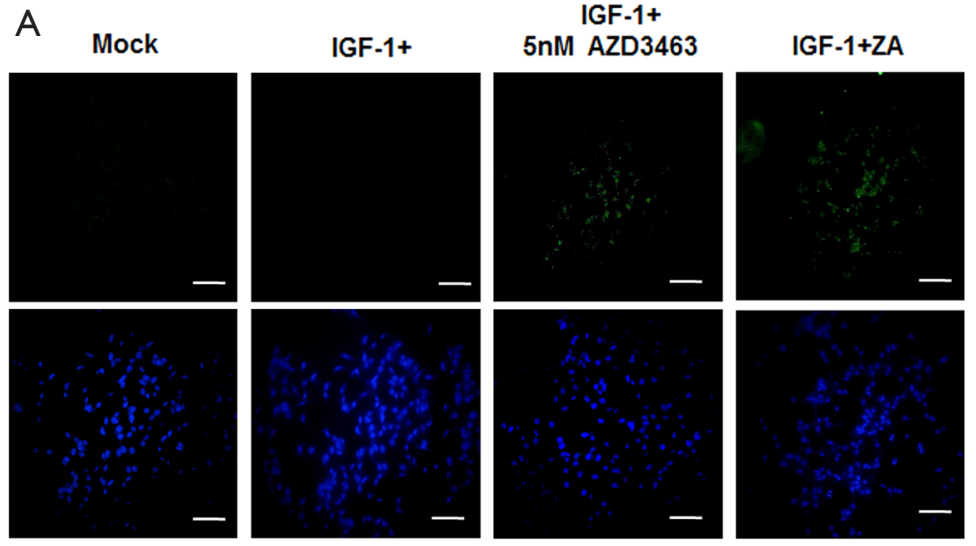

C
MW

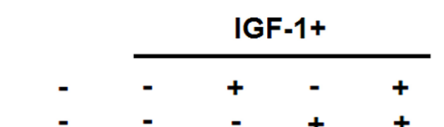

AZD 3463
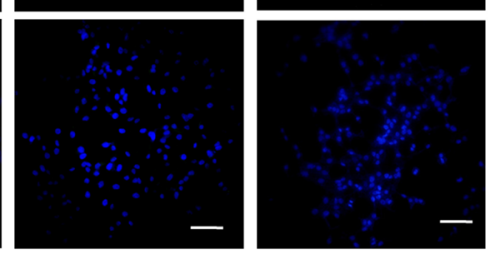

$95 \mathrm{kD}-$
$95 \mathrm{kD}-$
$60 \mathrm{kD}-$

IGF-1+ZA+

$5 \mathrm{nM}$ AZD3463
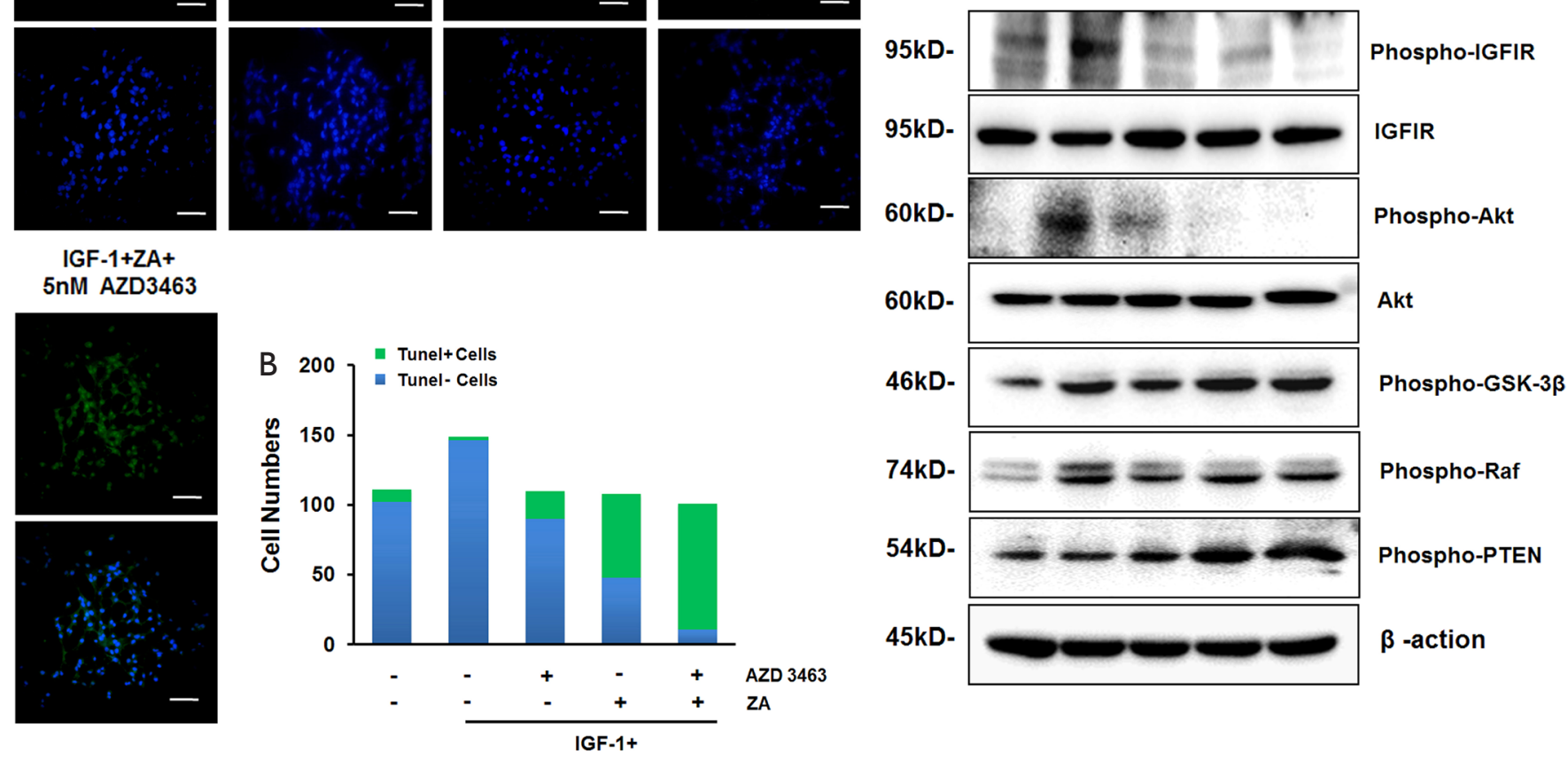

IGFIR

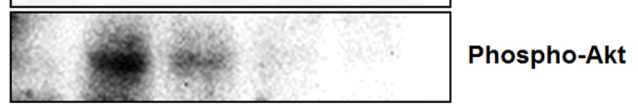

$60 \mathrm{kD}$

-Akt

kt

46kD

74kD-

Phospho-Raf

54kD-

$45 \mathrm{kD}$ -

$\beta$-action

Figure 4 AZD3463 effectively promoted the apoptosis of MDA-MB-213BO cells through blocking the PI3K-Akt signaling pathway. (A) TUNEL assay denoting the percentage of cell apoptosis in different experiment groups (green, TUNEL-positive cells); (B) quantification of viable and apoptotic MDA-MB-213BO cells; (C) Western blot assay showing the levels of phosphorylated and total IGF-1R, phosphorylated and total Akt, as well as phosphorylated GSK-3 $\beta$, Raf and PTEN in different experiment groups. $\beta$-actin was used as an internal control. Scale bar, $100 \mu \mathrm{m}$. PI3K-Akt, phosphoinositide 3-kinase; TUNEL, terminal deoxynucleotidyl transferase-mediated dUTP-biotin nick end labeling; IGF-1R, IGF-1 receptors; GSK-3 $\beta$, glycogen synthase kinase 3 beta; ZA, zoledronic acid; IGF-1, insulin-like growth factor I.

decrease in the bioluminescence signal compared to the control group (Figure 5C). However, neither compound was shown to be able to completely halt or even reverse the cancer progression as the bioluminescence signal intensities continued to rise slowly in both cases. In contrast, treatment with a combination of AZD3463 and ZA led to significant tumor shrinkage between week three and week five, demonstrating the best overall therapeutic effect among the four experiment groups $(\mathrm{P}<0.001)$ (Figure 5C). These findings lent convincing evidence that AZD3463 exhibited comparable or even greater antitumor activity against BM derived from breast cancer cells in the mouse model. Furthermore, the results suggested that the combined use of both compounds could serve as a promising therapeutic strategy for BM in breast cancer patients.

\section{Discussion}

The current study aims to evaluate the clinical value of AZD3463, an IGF-1R inhibitor, for combating the development and progression of BM in breast cancer patients. Initial studies indicated that AZD3463 could attenuate IGF-1-promoted phosphorylation of IGF-1R and concomitantly reduce the viability as well as metastatic potential of human breast adenocarcinoma MDA-MB$231 \mathrm{BO}$ cells in a dose-dependent manner. Subsequently, we demonstrated that $5 \mathrm{nM}$ of AZD3463 and $100 \mu \mathrm{M}$ of ZA produced similar effects on inhibiting IGF-1induced cell proliferation and promoting cell apoptosis. In comparison, cells treated with both compounds showed an even greater extent of apoptosis and lower level of 
A

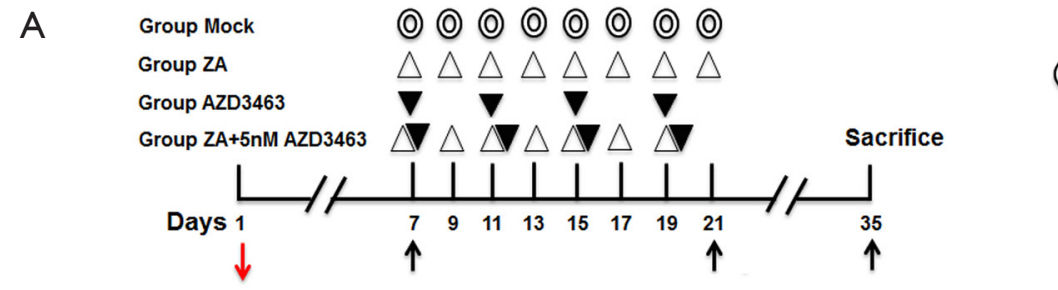

(0) PBS $\triangle$ ZA $\boldsymbol{\nabla}{ }_{A Z D 3463}$

B

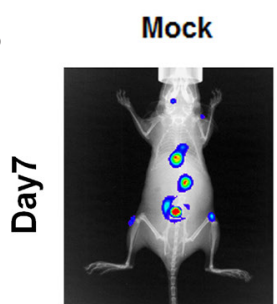

ZA
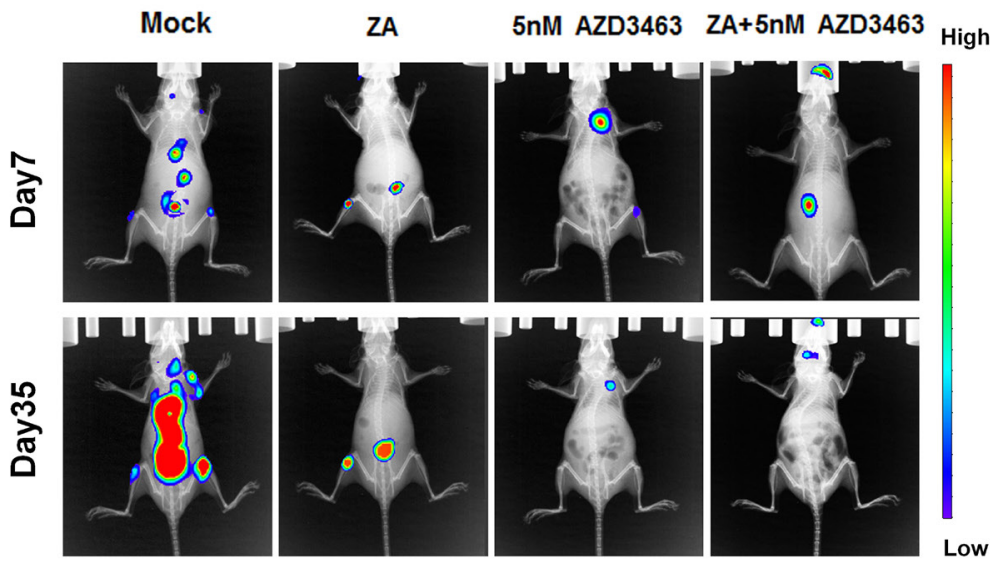

$\downarrow$ MDA-MB-231BO intracardiac injections

$\uparrow$ Bioluminescence imaging

D

Mock

ZA

5nM AZD3463

ZA+5nM AZD3463
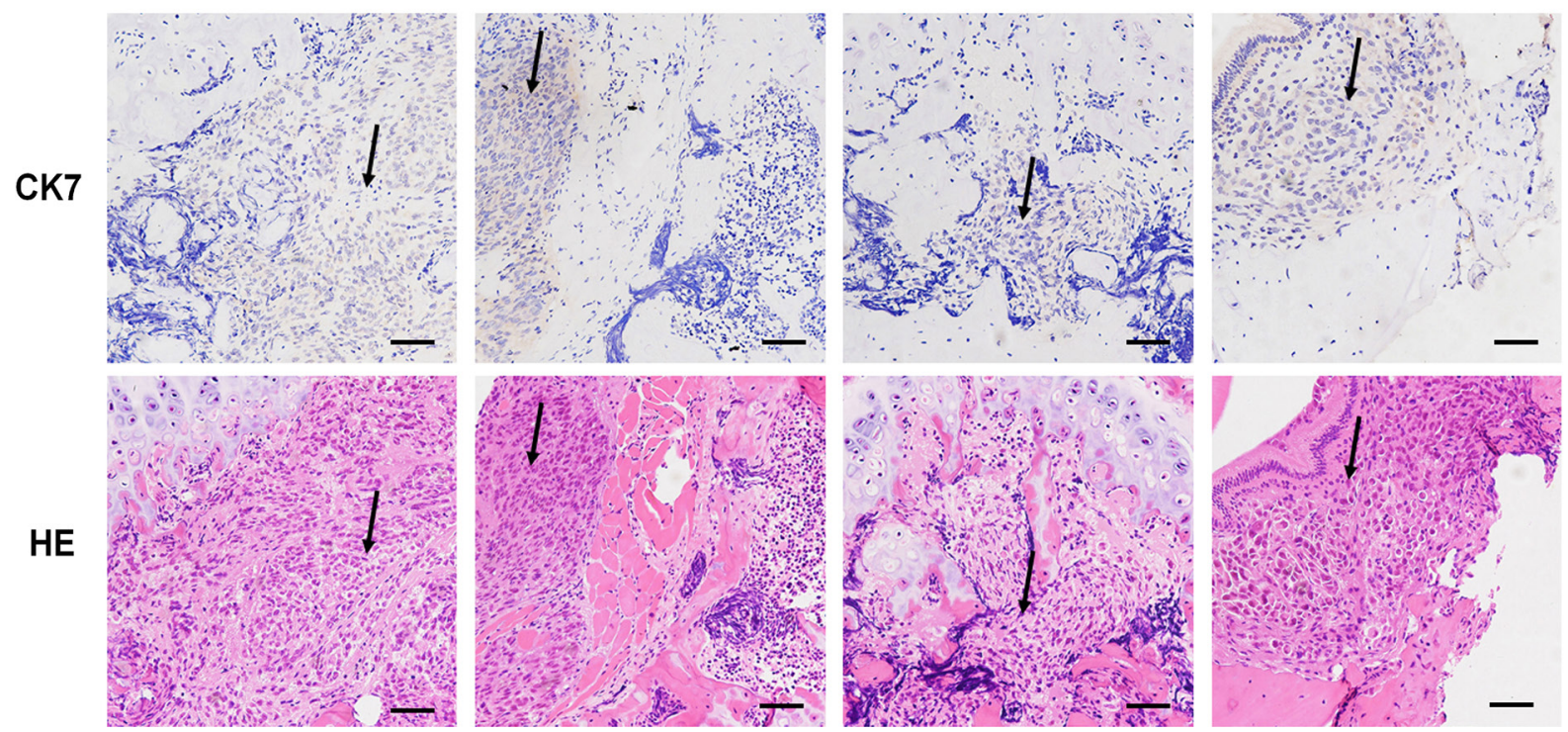

Figure 5 The combination of AZD3463 and ZA inhibited breast cancer metastasis to bone in murine model. (A) Treatment strategies for different experiment groups; (B) respective radiologic analysis merged bioluminescent images and X-ray radiography; (C) BLI quantitation of tumor load in different experiment groups ( $\mathrm{n}=6$ for each group) 1 week and 5 weeks after the inoculation of MDA-MB-213BO cells; (D) respective images of IHC analyses of CK7 as a marker of breast cancer (top) and H\&E (bottom) staining of bone tissue obtained from different experiment groups ( $\mathrm{n}=6$ for each group). The arrow indicated the invasive lesion in breast cancer. Scale bar, $100 \mu \mathrm{m} .{ }^{*}$, $\mathrm{P}<0.05$; ***, $\mathrm{P}<0.001$. ZA, zoledronic acid; BLI, bioluminescent imaging; H\&E, hematoxylin-eosin; PBS, phosphate-buffered saline; IHC, immunohistochemistry. 
proliferative capabilities. Quantitative analysis of a variety of factors involved in cell apoptosis revealed that AZD3463 could down-regulate the phosphorylation of Akt and increase that of PTEN, but exert no detectable effect on Raf or GSK-3 $\beta$. This suggested that IGF-1R modulated the apoptosis of MDA-MB-231BO cells by activating the PI3K-Akt signaling pathway. In a murine model of breast cancer metastasis to bone, the administration of AZD3463 exhibited similar or better therapeutic effect compared to ZA on slowing cancer development and progression. Most importantly, the combination of both AZD3463 and ZA not only demonstrated better antitumor activity compared to when only one of the two compounds was used, but also resulted in significant diminishment of the tumor lesions.

The metastasis of breast cancer to bone is associated with osteolysis, disruption of normal bone modeling and a variety of other pathological changes (28). IGF-1 is one of the most abundant factors in bone matrix and has been known to function as a key suppressor of cell apoptosis. The PI3KAkt pathway is considered to be the primary mechanistic route through which IGF-1 asserts its antiapoptotic effects. Less frequently, the regulatory role of IGF-1 in cell survival is manifested through the Raf-MEK-ERK signaling pathway (24). It is generally accepted that osteotropic migration of breast cancer is facilitated, under most circumstances, by an up-regulation of osteoclastic activity due to the discharge of soluble factors from the tumor cells. In turn, the destruction of bone tissues releases IGF1 and other mitogenic signaling molecules, which promote further tumor growth, adhesion and osteolysis (12). Due to its involvement in enhancing cell apoptosis, the regulation of the IGF-1R signaling pathway has been investigated as a potential therapeutic approach against metastatic tumors, particularly those in bone. Targeting of the IGF$1 \mathrm{R}$ pathway was found to augment the effectiveness of simvastatin, a stimulator of cell apoptosis, in the treatment of prostate cancer (29). However, it should be noted that clinical trials of IGF-1R inhibitors have yielded mixed results. A recent phase I study on the efficacy of an IGF-1R antibody CP-751,871 against nonhematologic cancers found limited evidence supporting the antitumor value of the drug (30). Another IGF-1R-targeting antibody, IMC A12, exhibited only moderate therapeutic activity when used in a monotherapy against metastatic castration-resistant prostate cancer (31). These disappointing findings possibly arose from the fact that the IGF-1R signaling pathway is located at a nexus point where a variety of different regulatory mechanisms converge and interact $(32,33)$.
Our finding that AZD3463 could significantly inhibit Akt phosphorylation is consistent with the results of several previous studies. Sampson and colleagues reported that AZD3463 could increase the cytotoxicity of SN-38, the active metabolite of irinotecan and topoisomerase 1 inhibitor, temozolomide and vorinostat in Ewing sarcoma tissues. This effect was attributed to the ability of AZD3463 to block the IGF-1R pathway, thereby suppressing the activity of Akt and STAT3, both of which could contribute to tumoral resistance to chemotherapy regimens (34). Similarly, AZD3463 was shown to effectively suppress the proliferation of neuroblastoma as a PI3K-Akt pathway inhibitor in a mouse xenograft model (17). In addition, AZD3463 demonstrated enhancing effect on the cytotoxicity of the antitumor drug doxorubicin on the cancer cells (17). Although there are only a few published studies on this topic, their results suggested that AZD3463 might achieve the best clinical efficacy when applied in conjunction with one or more additional chemotherapeutic drugs that target other oncogenic pathways to minimize the development of tumor resistance.

Other novel combination therapies that aim to inhibit both IGF-1R and additional targets have also been proposed with the aim to compensate for the reduction in IGF$1 \mathrm{R}$ pathway activity. For example, the use of an IGF1R inhibitor AZD9362 coupled with an Akt inhibitor AZD5363 demonstrated higher antitumor activity against estrogen receptor $\alpha$-positive breast cancer cells compared to when only one of the therapeutic agents was used (35), possibly due to the ability of the drug combination to block the compensatory stimulation of Akt that arose in the context of IGF-1R inhibition (36). In the current study, we also observed enhanced antitumor activity when ZA and AZD3463 were concurrently applied on metastatic breast cancer cells in bone. Multiple studies have indicated significantly diminished IGF-1 expression in bone tissues following the administration of $\mathrm{ZA}(37,38)$. It is possible that the observed therapeutic benefit of using both AZD3463 and $\mathrm{ZA}$ in our study could be attributed to the additional yet underexplored suppression of the IGF-1/IGF-1R axis provided by the bisphosphate compound. On the other hand, as stated earlier, the progression of osteolysis is a main contributing factor to the induction of tumor development in bone. In this regard, ZA-promoted down-regulation of osteoclastic activity has been linked to attenuated release of tumor-enhancing growth factors in the bone matrix, which could play a key role in its synergistic effect with AZD3463.

In summary, our study indicated that AZD3463 could effectively suppress the metastatic and proliferative 
capabilities of MDA-MB-231BO cells by modulating the PI3K-Akt signaling pathway, providing preliminary experimental evidence that supported the antitumor activity of AZD3463, particularly when administered together with $\mathrm{ZA}$, against BM derived from primary breast tumors. There would be a limitation that only one cell line was employed to study the anti-metastasis effect of AZD3463. Given that other widely-used BM cells were also derived from MDAMB-231 cells and the lack of breast cancer BM cell lines with hormone receptor (HR)-positive or human epidermal growth factor receptor 2 (HER2)-positive, future research could be required for the validation of therapeutic potential of AZD3463 in other preclinical mouse models.

\section{Acknowledgments}

The authors wish to thank for Lengfei Zhang, who provided purely technical help, and thank for Mofang Liu, who provided only general support. Lengfei Zhang and Mofang Liu are both from Institute of Biochemistry and Cell Biology, Shanghai Institutes of Biological Sciences, Chinese Academy of Sciences, 320 Yue Yang Road, Shanghai 200031 China. In addition, the authors wish to thank for AstraZeneca UK Limited (London, England) and Dr. Toshiyuki Yoneda (University of Texas Health Science Center at San Antonio, San Antonio, Texas, USA) to kindly provided AZD3463 and MDA-MB-231BO, respectively.

Funding: This work was supported in part by the National Natural Science Foundation of China (No. 81502267), the Key Medical Specialty Fund Projects of Shanghai (No. ZK2015A07), the 2019-2021 Shanghai Huangpu District Leading Medical Talent Training Plan (No. 2019LJ04), and the Shanghai Huangpu District Science Projects (No. HKQ201804).

\section{Footnote}

Conflicts of Interest: The authors have no conflicts of interest to declare.

Ethical Statement: The authors are accountable for all aspects of the work in ensuring that questions related to the accuracy or integrity of any part of the work are appropriately investigated and resolved. All animal experiments were performed in accordance with the National Institutes of Health guide for the care and use of laboratory animals (NIH Publications No. 8023, revised 1978) and approved by the Ethics Committee of Huangpu
Branch, Shanghai Ninth People's Hospital and Zhongshan Hospital, Fudan University (No. 2016ZSQZ54).

Open Access Statement: This is an Open Access article distributed in accordance with the Creative Commons Attribution-NonCommercial-NoDerivs 4.0 International License (CC BY-NC-ND 4.0), which permits the noncommercial replication and distribution of the article with the strict proviso that no changes or edits are made and the original work is properly cited (including links to both the formal publication through the relevant DOI and the license). See: https://creativecommons.org/licenses/by-nc-nd/4.0/.

\section{References}

1. Siegel RL, Miller KD, Jemal A. Cancer statistics, 2018. CA Cancer J Clin 2018;68:7-30.

2. Chen $\mathrm{W}$, Zheng R, Baade BD, et al. Cancer statistics in China, 2015. CA Cancer J Clin 2016;66:115-32.

3. Roodman GD. Mechanisms of bone metastasis. N Engl J Med 2004;350:1655-64.

4. Cetin K, Christiansen CF, Svaerke C, et al. Survival in patients with breast cancer with bone metastasis: a Danish population-based cohort study on the prognostic impact of initial stage of disease at breast cancer diagnosis and length of the bone metastasis-free interval. BMJ Open 2015;5:e07702.

5. Harries M, Taylor A, Holmberg L, et al. Incidence of bone metastases and survival after a diagnosis of bone metastases in breast cancer patients. Cancer Epidemiol 2014;38:427-34.

6. Svendsen ML, Gammelager H, Svaerke C, et al. Hospital visits among women with skeletal-related events secondary to breast cancer and bone metastases: a nationwide population-based cohort study in Denmark. Clin Epidemiol 2013;5:97-103.

7. Coleman RE. Metastatic bone disease: clinical features, pathophysiology and treatment strategies. Cancer Treat Rev 2001;27:165-76.

8. Wong MH, Stockler MR, Pavlakis N. Bisphosphonates and other bone agents for breast cancer. Cochrane Database Syst Rev 2012;15:CD003474.

9. Gnant M, Pfeiler G, Dubsky PC, et al. Adjuvant denosumab in breast cancer (ABCSG-18): a multicentre, randomised, double-blind, placebo-controlled trial. Lancet 2015;386:433-43.

10. Zheng H, Kang Y. Cradle of evil: osteogenic niche for early bone metastasis. Cancer cell 2015;27:153-5. 
11. Soki FN, Park SI, McCauley LK. The multifaceted actions of PTHrP in skeletal metastasis. Future Oncol 2012;8:803-17.

12. Mundy GR. Metastasis to bone: causes, consequences and therapeutic opportunities. Nat Rev Cancer 2002;2:584-93.

13. Onishi T, Hayashi N, Theriault RL, et al. Future directions of bone-targeted therapy for metastatic breast cancer. Nat Rev Clin Oncol 2010;7:641-51.

14. Hiraga T, Myoui A, Hashimoto N, et al. Bone-derived IGF mediates crosstalk between bone and breast cancer cells in bony metastases. Cancer Res 2012;72:4238-49.

15. Samani AA, Yakar S, LeRoith D, et al. The role of the IGF system in cancer growth and metastasis: overview and recent insights. Endocr Rev 2007;28:20-47.

16. Maki RG. Small is beautiful: insulin-like growth factors and their role in growth, development, and cancer. J Clin Oncol 2010;28:4985-95.

17. Wang Y, Wang L, Guan S, et al. Novel ALK inhibitor AZD3463 inhibits neuroblastoma growth by overcoming crizotinib resistance and inducing apoptosis. Sci Rep 2016;6:19423.

18. Jiang S, Zhang LF, Zhang HW, et al. A novel miR155/miR-143 cascade controls glycolysis by regulating hexokinase 2 in breast cancer cells. EMBO J 2012;31:1985-98.

19. Wang L, Zhang LF, Wu J, et al. IL-1betamediated repression of microRNA-101 is crucial for inflammation-promoted lung tumorigenesis. Cancer Res 2014;74:4720-30.

20. Miao Y, Zhang LF, Guo R, et al. (18)F-FDG PET/CT for Monitoring the Response of Breast Cancer to miR143-Based Therapeutics by Targeting Tumor Glycolysis. Mol Ther Nucleic Acids 2016;5:e357.

21. Zhang LF, Lou JT, Lu MH, et al. Suppression of miR199a maturation by HuR is crucial for hypoxia-induced glycolytic switch in hepatocellular carcinoma. EMBO J 2015;34:2671-85.

22. Yoneda T, Williams PJ, Hiraga T, et al. A bone-seeking clone exhibits different biological properties from the MDA-MB-231 parental human breast cancer cells and a brain-seeking clone in vivo and in vitro. J Bone Miner Res 2001;16:1486-95.

23. Coleman R, Cook R, Hirsh V, et al. Lipton. Zoledronic acid use in cancer patients: more than just supportive care? Cancer 2011;117:11-23.

24. Heidegger I, Pircher A, Klocker H, et al. Targeting the insulin-like growth factor network in cancer therapy.
Cancer Biol Ther 2011;11:701-7.

25. Surmeli Z, Gursoy P, Erdogan AP, et al. Combination of zoledronic acid and serine/threonine phosphatase inhibitors induces synergistic cytotoxicity and apoptosis in human breast cancer cells via inhibition of PI3K/Akt pathway. Tumour Biol 2016;37:3665-73.

26. Winter MC, Holen I, Coleman RE. Exploring the antitumour activity of bisphosphonates in early breast cancer. Cancer Treat Rev 2008;34:453-75.

27. Fragni M, Bonini SA, Bettinsoli P, et al. The miR-21/ PTEN/Akt signaling pathway is involved in the antitumoral effects of zoledronic acid in human breast cancer cell lines. Naunyn Schmiedebergs Arch Pharmacol 2016;389:529-38.

28. Chen YC, Sosnoski DM, Mastro AM. Breast cancer metastasis to the bone: mechanisms of bone loss. Breast Cancer Res 2010;12:215.

29. Nordstrand A, Lundholm M, Larsson A, et al. Wikstrom. Inhibition of the insulin-like growth factor-1 receptor enhances effects of simvastatin on prostate cancer cells in co-culture with bone. Cancer Microenviron 2013;6:231-40.

30. Haluska P, Shaw HM, Batzel GN, et al. Phase I dose escalation study of the anti insulin-like growth factor-I receptor monoclonal antibody CP-751,871 in patients with refractory solid tumors. Clin Cancer Res 2007;13:5834-40.

31. Hussain M, Rathkopf D, Liu G, et al. A randomised noncomparative phase II trial of cixutumumab (IMC-A12) or ramucirumab (IMC-1121B) plus mitoxantrone and prednisone in men with metastatic docetaxel-pretreated castration-resistant prostate cancer. Eur J Cancer 2015;51:1714-24.

32. King H, Aleksic T, Haluska P, et al. Can we unlock the potential of IGF-1R inhibition in cancer therapy? Cancer Treat Rev 2014;40:1096-105.

33. Chitnis MM, Yuen JS, Protheroe AS, et al. The type 1 insulin-like growth factor receptor pathway. Clin Cancer Res 2008;14:6364-70.

34. Sampson VB, Vetter NS, Kamara DF, et al. Vorinostat Enhances Cytotoxicity of SN-38 and Temozolomide in Ewing Sarcoma Cells and Activates STAT3/AKT/MAPK Pathways. PLoS One 2015;10:e0142704.

35. Fox EM, Kuba MG, Miller TW, et al. Autocrine IGF-I/ insulin receptor axis compensates for inhibition of AKT in ER-positive breast cancer cells with resistance to estrogen deprivation. Breast Cancer Res 2013;15:R55.

36. Cao L, Yu Y, Darko I, et al. Addiction to elevated insulin- 
like growth factor I receptor and initial modulation of the AKT pathway define the responsiveness of rhabdomyosarcoma to the targeting antibody. Cancer Res 2008;68:8039-48.

37. Perifanis V, Vyzantiadis T, Vakalopoulou S, et al.

Treatment of beta-thalassaemia-associated osteoporosis

Cite this article as: $\mathrm{Hu}$ GF, Wang C, Hu GX, Wu G, Zhang C, Zhu W, Chen C, Gu Y, Zhang H, Yang Z. AZD3463, an IGF-1R inhibitor, suppresses breast cancer metastasis to bone via modulation of the PI3K-Akt pathway. Ann Transl Med 2020;8(6):336. doi: 10.21037/atm.2020.02.110 with zoledronic acid. Br J Haematol 2004;125:91-2.

38. Perifanis V, Vyzantiadis T, Tziomalos K, et al. Effect of zoledronic acid on markers of bone turnover and mineral density in osteoporotic patients with beta-thalassaemia. Ann Hematol 2007;86:23-30. 